\title{
Elaborar mapas conceptuales con la Cmaptools en el proceso enseñanza aprendizaje
}

\author{
M. Yolanda González-Alonso ${ }^{a}$, Montserrat Santamaría-Vázquez ${ }^{\mathrm{a}}$ y Valeriana Guijo- \\ Blanco $^{a}$
}

${ }^{a}$ Universidad de Burgos. Facultad de Ciencias de la Salud. Paseo Comedadores, s/n 09001 Burgos. mygonzalez@ubu.es, msvazquez@ubu.es y vguijo@ubu.es

\begin{abstract}
The innovation in the teaching-learning process uses the new technologies which allow the student to take an active role in their knowledge construction. In this study, it is analyzed the efficacy of concept maps, built with the Cmaptool as a didactic strategy in first course of the occupational therapy degree. Students of the subject of Psychology built concept maps, which are evaluated using a rubric that assesses different aspects as the content, the appearance, the accuracy and the organization. At the end of the semester, students evaluated the effect of the use of this tool in their own learning through an ad-hoc questionnaire. Afterwards the quality of the map and the student's opinion were related with the student academic performance. It was found no relation between the variables.

Although there are evidences that show the efficacy of the concept maps in the teaching-learning process and that they can improve the comprehension of complex and interrelated subjects, it is necessary to motivate the students in order to they understand the creation of a concept map as a reflexive process but not only a mechanic one. Likewise it is necessary to provide constant feedback and specific teaching about the tool.
\end{abstract}

Keywords: meaningful learning, knowledge representation, educational innovation, performance, new technologies

\footnotetext{
Resumen

La innovación en los proceso de enseñanza-aprendizaje aprovecha las nuevas tecnologías que permiten al estudiante tomar un papel activo en la construcción de su conocimiento. En este estudio se analiza la eficacia de los mapas conceptuales, elaborados a través de la herramienta Cmaptool como estrategia didáctica de primer curso del grado de terapia ocupacional. En la asignatura Psicología, crean mapas conceptuales que son evaluados a través de una rúbrica que contempla aspectos tales como el contenido, la apariencia, la precisión y la organización. Al finalizar el semestre el alumnado evaluó la repercusión del uso de esta herramienta en su propio aprendizaje a través de un cuestionario ad-hoc. Se relacionó la calidad
} 
del mapa y la opinión del estudiante con su rendimiento académico, no encontrándose relaciones entre las variables.

Se concluye que aunque hay evidencias que demuestran la eficacia de los mapas conceptuales en el proceso de enseñanza-aprendizaje y que pueden mejorar la comprensión de temas complejos e interrelacionados es necesario para que esto ocurra motivar al alumnado para que entienda la construcción del mapa como un proceso reflexivo y no un simple proceso mecánico, así mismo se necesita ofrecer un feedback constante y un adiestramiento específico en la herramienta.

Palabras clave: aprendizaje significativo, la representación del conocimiento, innovación docente, el rendimiento, nuevas tecnologías.

\section{Introducción}

El interés del profesorado por mejorar la docencia y el aprendizaje del alumnado alienta experiencias educativas innovadoras que integran aplicaciones informáticas en las metodologías activas. Un buen ejemplo lo constituyen la elaboración de mapas conceptuales que han tomado un gran auge en los últimos años por el desarrollo de herramientas informáticas que facilitan su confección, entre ella destaca la Cmaptools.

En un mapa conceptual se plasma un resumen esquemático, ordenado y jerarquizado de los aprendizajes de los alumnos. Los mapas conceptuales se han venido utilizando en diferentes niveles educativos y en diversas materias del currículum con eficacia probada (Cañas y Novak, 2006).

Los mapas conceptuales constituyen una estrategia de enseñanza-aprendizaje caracterizada por estar centrada en el estudiante. Se apoyan en los planteamientos de Ausubel (1998, 2002) en lo referente a la evolución de las ideas previas que poseen los estudiantes para construir un nuevo conocimiento y en los de Novak (2002) sobre cómo los conceptos se relacionan entre sí y constituyen una organización jerárquica.

El proceso de elaboración de un mapa conceptual constituye una herramienta de aprendizaje útil para el alumnado porque exige profundizar en contenidos, relacionar significativamente conceptos y fomentar el desarrollo integral del estudiante (Cañas y Novak, 2006).

Los mapas conceptuales pueden utilizarse bien como recurso comunicativo del profesor para mostrar la información sobre un tema o bien como instrumento de representación del conocimiento logrado por un alumno o por un grupo si se utiliza como un recurso colaborativo Martín-Salinas, Parro-Moreno y Cid-Galán (2015).

En esta investigación los estudiantes aprendieron contenidos de una asignatura y los plasmaron en mapas conceptuales. La realización de mapas conceptuales permitió una representación global de cada tema de estudio por medio de una organización jerárquica a través de una relación de generalización y especialización entre los conceptos (Gallego,

(cc) EY-NC-ND 2016, Universitat Politècnica de València

Congreso IN-RED (2016) 
Crisol, y Gámiz, 2013). Esto pretendía motivar al estudiante y mantener el interés por la asignatura a la vez que se adquirían los objetivos marcados en la guía didáctica.

Para el alumno es una herramienta fundamental que supone aprender significativamente aunque también requiere unas buenas bases (Novak, 1998) como tener unos conocimientos previos relevantes, relacionarlos con otros conceptos y estar motivado por este tipo de aprendizaje e interés en buscar la relación entre los conceptos.

La elaboración de un mapa conceptual consiste en realizar una representación gráfica que permita tener una percepción inmediata del tema propuesto de forma categorizada, ordenada y comprensible. Esta tarea pone de manifiesto el razonamiento seguido por el alumno para establecer las distintas conexiones, que a su vez reflejan la profundidad con la que han tratado los temas y la capacidad de interrelación. Igualmente permite valorar la capacidad de deducción del alumno.

Gracias a las nuevas tecnologías, hoy existen numerosos programas para crear mapas conceptuales de manera clara y sencilla que pretenden motivar y ayudar a los estudiantes. En la web se pueden encontrar herramientas gratuitas para crear mapas conceptuales entre las que destaca la Cmaptools, se trata de un software desarrollado por el Institute for Human and Machine Cognition (IHNC, 2005) de la Universidad de West Florida (Estados Unidos). Es una herramienta fácil de usar y tiene funciones útiles en el campo educativo (Chrobak, García y Prieto, 2015; Murga-Menoyo, Bautista y Novo, 2011) y en la investigación (Pontes, Serrano, Muñoz y López, 2011).

\section{Objetivos}

Las propuestas innovadoras han de ser analizadas para comprobar su efecto en el proceso de aprendizaje, conscientes de ello se planteó el siguiente objetivo general: Analizar la utilidad del uso de mapas conceptuales con apoyo de la Cmaptools para los procesos de enseñanza aprendizaje en la asignatura de Psicología, desde la opinión de los alumnos de primero de grado de Terapia Ocupacional.

Este objetivo se concreta en los siguientes objetivos específicos:

- Desarrollar las competencias necesarias para manejar el software Cmaptools en la realización de mapas conceptuales.

- Establecer la calidad de los mapas conceptuales evaluados a través de una rúbrica.

- Estimar la valoración del alumnado de la actividad.

- Determinar la relación entre la valoración de los mapas conceptuales y el rendimiento global en la asignatura.

\section{Desarrollo de la innovación}

La experiencia de innovación docente que se presenta se realizó en la asignatura de Psicología, que se imparte en el Grado de Terapia Ocupacional, en la Universidad de Burgos. Se trata de una asignatura obligatoria que se imparte en el segundo semestre del

(cc) BY-NC-ND 2016, Universitat Politècnica de València

Congreso In-Red (2016) 
primer curso. Cada alumno debía presentar al menos dos mapas elaborados individualmente.

En una sesión de clase práctica, al inicio del curso, se les prepara para que aprendan a diseñar y construir los mapas conceptuales, para ello se siguen diferentes pasos:

a) Explicación de qué es un mapa conceptual y por qué utilizarlos.

- El valor de los mapas conceptuales como instrumento de jerarquización y estructuración de los conocimientos explicados.

- Motivación, por su valor como resumen visual que facilita contextualizar el contenido dentro del tema de estudio facilitando la interrelación del conocimiento, la memorización con significado y el recuerdo duradero.

b) Explicación de la herramienta de acceso gratuito Cmaptools (http://cmap.ihmc.us /cmaptools/).

- Descarga de la página oficial.

- Uso de Cmaptools.

- Ejemplos de "buenos mapas conceptuales".

c) Presentación de la rúbrica con la que evaluarán los mapas elaborados por el alumnado.

Para evaluar la calidad de los mapas conceptuales se ha elaborado una rúbrica (tabla 1) que atiende a los siguientes criterios: a) contenido, trata el tema en profundidad con conceptos, características y ejemplos, con un conocimiento excelente del tema; b) apariencia, la organización es fácil de interpretar, contiene todos los conceptos claves y la información necesaria para entenderlo, todo gira alrededor de un tema; c) precisión, la terminología y notas son correctas haciendo fácil y atractiva la comprensión del contenido, sin faltas de ortografía ni de puntuación; y d) organización, el contenido está bien organizado de acuerdo a su nivel de importancia, todos los conceptos tienen relaciones adecuadas incluso conexiones complejas que sugieren comprensión profunda del material.

Tabla 1. Rúbrica para evaluar un mapa conceptual

\begin{tabular}{|c|c|c|c|c|}
\hline CRITERIOS & NIVEL 3 & NIVEL 2 & NIVEL 1 & NIVEL 0 \\
\hline CONTENIDO & $\begin{array}{l}\text { Trata el tema en profundidad con } \\
\text { conceptos, caracteristicas y ejemplos. } \\
\text { El conocimiento del tema es excelente. }\end{array}$ & $\begin{array}{l}\text { Incluye conocimientos básicos sobre el } \\
\text { tema. }\end{array}$ & $\begin{array}{l}\text { Incluye información esencial sobre } \\
\text { el tema perotiene } 1-2 \text { errores. }\end{array}$ & $\begin{array}{l}\text { El contenido es mínimo y } \\
\text { tiene varios errores. }\end{array}$ \\
\hline APARIENCIA & $\begin{array}{l}\text { La organización es fácil de interpretar. } \\
\text { Contiene todos los conceptos claves y } \\
\text { la información necesaria para } \\
\text { entenderlo. } \\
\text { El mapa gira alrededor de un concepto } \\
\text { o tema. }\end{array}$ & $\begin{array}{l}\text { Coloca la mayoría de los conceptos de } \\
\text { manera adecuada, estableciendo } \\
\text { relaciones apropiadas la mayoría de } \\
\text { las veces con una interpretación fácil. } \\
\text { Contiene buena cantidad de conceptos } \\
\text { claves e información necesaria. } \\
\text { El mapa gira alrededor de un concepto } \\
\text { o tema. }\end{array}$ & \begin{tabular}{|l|} 
Coloca algunos conceptos de \\
manera adecuada pero pobre y con \\
pocas relaciones. \\
Las relaciones están correctas pero \\
la jerarquía confundida.
\end{tabular} & $\begin{array}{l}\text { Hay jerarquía pero no en el } \\
\text { orden correcto. No está bien } \\
\text { clasificada la información. Los } \\
\text { conceptos sin relación }\end{array}$ \\
\hline PRECISIÓN & $\begin{array}{l}\text { La terminología y notas son correctas } \\
\text { haciendo fácil y atractiva la } \\
\text { comprensión del contenido. } \\
\text { No tiene faltas de ortografía, ni de } \\
\text { puntuación. }\end{array}$ & $\begin{array}{l}\text { La mayoría de la terminología y notas } \\
\text { son correctas. } \\
\text { Tiene pocas faltas de ortografía y/o } \\
\text { puntuación. }\end{array}$ & $\begin{array}{l}\text { Usa poco la terminología y algunas } \\
\text { veces no es fácil entender el } \\
\text { contenido. } \\
\text { Tiene algunas faltas de ortografía y } \\
\text { de puntuación. }\end{array}$ & \begin{tabular}{|l} 
Utiliza poco o \\
inapropiadamente la \\
terminología y las notas. \\
Tiene muchas faltas de \\
ortografía y puntuación.
\end{tabular} \\
\hline ORGANIZACIÓN & $\begin{array}{l}\text { Contenido bien organizado de acuerdo } \\
\text { a su nivel de importancia, desde el más } \\
\text { general al más específico. } \\
\text { Todos los conceptos tienen conexiones } \\
\text { o relaciones adecuadas. } \\
\text { Hay conexiones complejas que } \\
\text { sugieren una comprensión profunda } \\
\text { del material con claridad excelente. }\end{array}$ & $\begin{array}{l}\text { El mapa es claro y permite } \\
\text { comprender la mayor parte de la } \\
\text { temática. } \\
\text { La mayoría de los conceptos tienen } \\
\text { conexioneso relaciones adecuadas y } \\
\text { correctamente establecidas. } \\
\text { Hay algunas conexiones complejas que } \\
\text { sugieren comprensión profunda del } \\
\text { tema. }\end{array}$ & \begin{tabular}{|l|} 
La organización en conjunto \\
aparenta debilidad. La mayor parte \\
del contenido está organizado \\
lógicamente y permite comprender \\
algunas partes de la temática. \\
Aunque también aparecen \\
conexioneso relaciones \\
inadecuadas o incorrectas.
\end{tabular} & $\begin{array}{l}\text { La organización no es clara o } \\
\text { lógica. Hay solo muchos } \\
\text { hechos. } \\
\text { El contenido se entiende con } \\
\text { dificultad, debido a la falta de } \\
\text { coherenciay claridad. } \\
\text { Hay conexiones triviales que } \\
\text { sugieren poca o nula } \\
\text { comprensión de la materia. }\end{array}$ \\
\hline
\end{tabular}

(c)) BY-NC-ND 2016, Universitat Politècnica de València 
Toda la información se deja a disposición del alumnado en la página web de la asignatura.

A lo largo del curso la profesora utiliza mapas conceptuales para explicar algunos de los contenidos, en concreto los relativos a los contextos de desarrollo y a la etapa de la adolescencia.

Al finalizar el curso todos los estudiantes contestan un cuestionario con siete preguntas para conocer la opinión del encuestado, 4 de los ítems dirigidos a valorar la opinión respecto al trabajo realizado con los mapas conceptuales como herramienta de aprendizaje y 3 dirigidos a valorar el uso de soportes informáticos como herramienta facilitadora. Los ítems se valoran de acuerdo a una escala tipo Likert de 10 opciones para las respuestas donde 1 es nada de acuerdo y 10 totalmente de acuerdo.

\section{Resultados}

En lo referente a los mapas conceptuales realizados por los alumnos, éstos contienen gran cantidad de conceptos, en general relevantes, dispuestos en varios niveles jerárquicos. Los mapas conceptuales que se presentan han sido realizados correctamente y muestran los contenidos según los criterios que corresponden a un adecuado nivel de conocimiento. Las diferencias entre las puntuaciones obtenidas por los alumnos no alcanzan nivel de significatividad.

Sin embargo, a lo largo del proceso algunos de ellos se quejan de dificultades para utilizar el Cmaptools y piden entregarlos elaborados a mano.

El nivel de aprobados es relativamente alto, ya que un $76 \%$ supera el examen en la primera convocatoria, esto supuso un $10 \%$ más que los alumnos del curso anterior.

En cuanto a la valoración de la utilización de los mapas conceptuales y la herramienta los datos son los que se recogen en la tabla 2 .

Tabla 2. Promedio de resultados

\begin{tabular}{|c|c|c|c|c|c|c|}
\hline ÍTEMS & Mín. & Máx. & Media & DT & Moda & Med. \\
\hline 1. Me han servido para organizar los contenidos & 1 & 10 & 5,41 & 2,63 & 7 & 6 \\
\hline 2. Me ha ayudado a aprender & 1 & 10 & 5,22 & 2,45 & 7 & 6 \\
\hline $\begin{array}{l}\text { 3. Me han servido para darme cuenta de la } \\
\text { relación entre los contenidos explicados }\end{array}$ & 1 & 9 & 5,52 & 2,05 & 7 & 6 \\
\hline $\begin{array}{l}\text { 4. Es una estrategia de aprendizaje válida para el } \\
\text { estudio de cualquier asignatura }\end{array}$ & 2 & 10 & 6,07 & 2,16 & 7 & 6 \\
\hline $\begin{array}{l}\text { 5. La mayor dificultad ha sido tener que utilizar el } \\
\text { software cmaptools }\end{array}$ & 1 & 10 & 5,31 & 3,41 & 1 & 5 \\
\hline $\begin{array}{l}\text { 6. La utilización de las tecnologías de la } \\
\text { información supone un gran problema para mí }\end{array}$ & 1 & 10 & 4,04 & 2,65 & 1 & 3,5 \\
\hline $\begin{array}{l}\text { 7. No creo necesario la utilización de nuevas } \\
\text { tecnologías como estrategia de aprendizaje } \\
\text { para el estudio y asimilación de los contenidos }\end{array}$ & 1 & 10 & 4,11 & 2,60 & $1^{\mathrm{a}}$ & 3 \\
\hline
\end{tabular}

2016, Universitat Politècnica de València

Congreso In-Red (2016) 
Se incluye en la tabla los valores mínimos y máximos y los valores de las medidas de tendencia central ya que se en este caso, las medias ofrecen poca claridad para el análisis de los datos. La mediana y especialmente la moda, se considera que visualizan mejor la valoración de cada uno de los ítems por los alumnos. Se puede deducir, de la lectura de los datos, que los alumnos valoran positivamente la utilización de los mapas conceptuales ya que les ayuda para organizar, jerarquizar y aprender y sin embargo rechazan el uso de una herramienta específica.

Se estudió si se daba algún tipo de correlación entre las respuestas a los ítems encontrándose diferentes niveles de significatividad. Opinar que los mapas les han ayudado a organizar los contenidos correlaciona positivamente con me ha ayudado a aprender $(\mathrm{r}$ $, 770 ; \mathrm{p}=, 000)$ y con darme cuenta de la relación entre los contenidos $(\mathrm{r}, 832 ; \mathrm{p}=, 000)$ y con es una estrategia válida para el estudio de cualquier asignatura $(\mathrm{r}, 343 ; \mathrm{p}=, 020)$. El ítem me ayuda a aprender mantiene también una correlación significativa con los otros que valoran la utilizada de los mapas conceptuales: correlación ,748 (p= ,000) y ,444 (p= ,002) respectivamente. También el ítem 3, (me) ha servido para darme cuenta de (observar) la relación entre los contenidos explicados alcanza un buen nivel de correlación con es una estrategia válida para el estudio de cualquier asignatura $(\mathrm{r}, 397 ; \mathrm{p}=, 006)$.

Los datos sobre el valor de los mapas en el proceso de aprendizaje están en consonancia con los encontrados por Martín-Salina, Parro-Moreno y Cid-Galán (2015) con un grupo de estudiantes de enfermería. De ellos se puede concluir que realizar mapas conceptuales de los diversos temas abordado en una asignatura les va a permitir ver la relación entre los conceptos y jerarquizarlos.

Respecto a la valoración de la aplicación los resultados también indican niveles de correlación altos y significativos entre las diferentes medidas. Los alumnos que indican que la mayor dificultad ha sido tener que utilizar Cmap coinciden en que las tecnologías de la información son un problema para ellos $(\mathrm{r}, 672 ; \mathrm{p}=, 000)$ o que no creen necesario la utilización de nuevas tecnología ( $\mathrm{r}, 469 ; \mathrm{p}=, 001)$, en la misma línea la correlación entre los ítems 6 y 7 es positiva y significativa $(r, 405 ; p=, 006)$.

Algunas investigaciones han dejado constancia de la dificultad de los alumnos en la realización de los mapas (Domínguez y Manzano, 2012) debido a los contenidos, a la necesidad de selección o a la superposición de tareas. Sin embargo, en este caso aparece un fuerte rechazo a la herramienta que consideramos ha podido influir en el proceso. No deja de sorprender que alumnos que se consideran digitales indique que la mayor dificultad ha sido el uso de una aplicación, que está suficientemente probada y que puede facilitar la realización del mapa. No se indagó sobre las razones del rechazo pero la reflexión ante los datos y los comentarios de los alumnos a lo largo del curso ha llevado a pensar que la formación ofrecida por los docentes había sido escasa y el grado de ansiedad del alumno al enfrentarse a la tarea y a la aplicación había sido elevada.

El objetivo último que se espera al implementar una propuesta metodológica es favorecer el aprendizaje de los alumnos y que esto se refleje en la evaluación y que los alumnos sean capaces de establecer esa relación. Sin embargo en este caso, las correlaciones encontradas

(c)) BY-NC-ND 2016, Universitat Politècnica de València

Congreso IN-RED (2016) 
entre las calificaciones en el examen, en la primera convocatoria y la valoración de los mapas conceptuales no ha logrado niveles de significatividad salvo con el ítem 7, no creo necesario la utilización de nuevas tecnologías como estrategia de aprendizaje para el estudio y asimilación de los contenido. En este caso la correlación es negativa 3, 359 ( $\mathrm{p}=$ ,015) por lo que parece que aquellos alumnos que tienen mejor rendimiento académico valoran más negativamente el uso de la aplicación. A la vista de los resultados se puede concluir que el grupo ha realizado un buen nivel de aprendizaje y que los alumnos valoran la utilización de los mapas conceptuales como recurso de aprendizaje, sin embargo no se ha llegado a constatar que se dé una relación directa entre el rendimiento y una buena construcción de mapas conceptuales. Este resultado no esperado supone una diferencia con otros estudios como los de Chiu (2008) o Gallego, Crisol y Gámiz (2013).

\section{Conclusiones}

La utilización de mapas conceptuales en la docencia universitaria pretende contribuir al aprendizaje significativo del alumnado pero es necesario comprobar la efectividad de las innovaciones, ya que factores personales, del contenido o de la organización docente pueden hacer que no se logren los resultados esperados. Por eso, la intención de los profesores implicados en el estudio fue comprobar si la realización de mapas conceptuales de los contenidos de una materia consiguió mejorar la asimilación de los estudiantes. Este estudio ha permitido comprobar los resultados de integrar en el proceso de enseñanza aprendizaje de alumnos universitarios un recurso didáctico, los mapas conceptuales.

La experiencia se puede considerar positiva aunque se considera necesario mantener a lo largo de los cursos está metodología para que los alumnos "automaticen" su uso y no consideren un recurso, solo para alguna de las materias de estudio.

El rechazo a la herramienta requiere que se siga investigando sobre este tema, para confirmar si es una respuesta particular de un grupo o se repite en otros grupos. Para asegurar una mejor acogida se considera conveniente, tras este estudio, sistematizar mejor y realizar alguna práctica de uso del Cmaptools en presencia del profesor o de alumnos expertos que puedan solucionar las dudas que surjan.

\section{Referencias}

AUSUBEL, D.P. (2002) Adquisición y retención del conocimiento. Una perspectiva cognitiva. España: Paidós.

CHIOU, C.C. (2008). The effect of concept mapping on students' learning achievements and interest. Innovations in Education and Teaching International, 45, 4, 375-387.

CHROBAK, R., GARCÍA SEMPERE, P. y PRIETO, A.B. (2015). Creatividad, mapas conceptuales y TIC en educación. EDMETIC, Revista de Educación Mediática y TIC, 4(1), 78-94.

(c)) EY-NC-ND 2016, Universitat Politècnica de València

Congreso In-Red (2016) 
DOMÍNGUEZ MARRUFO, L.S. y MANZANO CAUDILLO, J. (2012). Mastery and Appropriation of Concept Mapping in Higher Education. En A.J. Cañas, J.D. Novak y J. Vanhear (Eds.) Concept Maps: Theory, Methodology, Technology. Proceedings of the Fifth International Conference on Concept Mapping. Malta, 345-352.

IHMC (2005). CMapTools v.4 [aplicación informática]. USA: Institute for Human and Machine Cognition. http://cmap.ihmc.us/docs/theory-of-concept-maps-spanish.php

MARTÍN-SALINAS, C., PARRO-MORENO, I. y CID-GALÁN, M.L. (2015). Utilización de mapas conceptuales como innovación docente para el desarrollo de competencias. Educación Médica, 16 (3), 173-176.

MURGA-MENOYO, M.A, BAUTISTA, M.J. y NOVO, M. (2011). Mapas conceptuales con Cmap Tools en la enseñanza universitaria de la educación ambiental. Estudio de caso en la Uned. Enseñanza de las Ciencias, 29 (1), 47-60.

NOVAK, J.D. (2002) Meaningful Learnimg: The Essential Factor for Conceptual Change in Limited or Inappopriate Propositional Hierarchies Leading to empowerment of Learniers. Science Education, 4 (86), 548-571.

NOVAK, J.D. (1998). Conocimiento y aprendizaje. Los mapas conceptuales como herramientas facilitadoras para escuelas y empresas. Madrid: Alianza.

NOVAK, J.D. y CAÑAS, A.J. (2006). La Teoría Subyacente a los Mapas Conceptuales y a Cómo Construirlos. Technical Report IHMC CmapTools 2006-01, Florida Institute for Human and Machine Cognition (IHMC). Accesible en http://cmap.ihmc.us/Publications/ $\underline{\text { ResearchPapers/TeoriaCmaps/TeoriaSu byacenteMapasConceptuales.html }}$

PONTES, A., SERRANO, R., MUÑOZ, J.M. y LÓPEZ, I. (2011). Innovación educativa sobre aprendizaje colaborativo con Cmap Tools en la formación inicial docente. Revista Iberoamericana de Evaluación Educativa, 4(2), 136-154. 